\title{
LA ESCUELA SECUNDARIA ARGENTINA (1960-2010). FUERZAS Y TENSIONES EN SUS PRÁCTICAS DE ENSEÑANZA
}

\author{
Fernando Cazas* \\ www.uba.ar
}

Resumen: El presente artículo aborda algunos aspectos del desarrollo de la escuela secundaria argentina durante parte del siglo XX y los inicios del XXI. Pone el foco en ciertos cambios y también en ciertas continuidades que se han dado, atravesando los distintos intentos de reformas al nivel. El autor identifica algunas fuerzas que operan detrás de estos cambios y continuidades generando tensiones sobre las formas de enseñar.

Palabras claves: Escuela secundaria; Argentina; Democratización; Enseñanza; Reformas.

\section{THE ARGENTINE SECONDARY SCHOOL (1960-2010). STRENGTHS AND TENSIONS IN PRACTICES TEACHING}

\begin{abstract}
This article addresses some aspects of the development of Argentine secondary school during part of the twentieth century and the beginnings of the XXI. It focuses on certain changes and also on certain continuities that have occurred, going through different attempts at reform at the level. The author identifies some forces operating behind these changes and continuities creating tensions over the ways of teaching.
\end{abstract}

Keywords: High school; Argentina; Democratization; Teaching; Reforms.

$$
\text { * } * *
$$

\footnotetext{
* Máster en Ciencias Sociales. Docente en la Universidad de Buenos Aires, UBA (Argentina). Contacto: fernando cazas@yahoo.com.ar.
} 


\section{Presentación}

La escuela secundaria argentina, ha experimentado muchas reformas desde sus inicios. Si bien todas estas coinciden mayormente en las críticas a la escuela secundaria tradicional, sus propuestas de modelos superadores varían notoriamente. Hay coincidencia en el diagnóstico, pero no en las soluciones propuestas. Incluso, estas suelen ser casi opuestas en algunos casos. La sucesión de reformas de la escuela secundaria (en especial a partir de la década del ‘60), no resulta inocua sobre las prácticas de enseñanza. A continuación, un recorrido que intentará avanzar sobre la comprensión de las fuerzas y tensiones detrás de cada intento de reforma.

\section{El modelo del bachillerato francés}

La escuela secundaria o media argentina (utilizaremos ambas denominaciones como equivalentes a lo largo del presente artículo) ha estado en el foco de las propuestas reformistas casi desde los albores de la conformación del sistema educativo nacional. Ya hacia fines del siglo XIX, el entonces ministro del área Osvaldo Magnasco proponía una primera reforma de las escuelas medias para adecuarlas a los nuevos tiempos que se avecinaban. La escuela secundaria argentina nació al amparo del modelo de bachillerato francés, admirado por las clases pudientes de Buenos Aires y también por las aristocracias terratenientes de las provincias. Un bachillerato humanista y enciclopedista que formaba hombres cultos para integrar la elite dirigente del país. Magnasco criticaba ese modelo y miraba con buenos ojos a los países anglosajones (Alemania o EE. UU.) que daban lugar a las ciencias naturales y a la moderna tecnología en las aulas de sus escuelas secundarias. La idea era cerrar algunos de los bachilleratos existentes y en su reemplazo crear escuelas para la enseñanza de oficios técnicos que sería útiles para el desarrollo de la eco- 
nomía nacional. El proyecto del ministro chocó con la negativa de un parlamento nacional, cuyos legisladores eran mayormente egresados del criticado bachillerato. La iniciativa de Magnasco no pudo concretarse (TIRAMONTI; SUSANBAR, 2015).

Para principios del siglo XX, Saavedra Lamas (a la sazón nuevo ministro de instrucción pública), presenta una nueva propuesta reformista. La idea del ministro era crear una escuela intermedia ubicada entre la escuela primaria y la secundaria. Esta propuesta se basaba, entre otras cuestiones, en novedosos postulados de la moderna psicología. Para algunos autores (Dussel, 2006) no faltan motivos para pensar que está reforma estuvo inspirada en las ideas de Víctor Mercante' ${ }^{1}$ La escuela intermedia daría cabida a los púberes y adolescentes, generando un nivel con características apropiadas para estas edades.

A pesar de los argumentos hasta aquí expuestos, no es descabellado pensar que tanto Magnasco como Saavedra Lamas, habían percibido que las nuevas clases medias argentinas (en especial las de Buenos Aires y las grandes capitales provinciales), pujaban porque sus hijos ingresaran a la escuela secundaria. A estas escuelas ${ }^{2}$ habían concurrido tradicionalmente

${ }^{1}$ Comenta Dussel (2006) sobre las ideas de Víctor Mercante: "La propuesta buscaba crear una "escuela intermedia" de tres años entre los niveles primario y secundario. Esta escuela intermedia incluiría dos ejes centrales: literario y científico, por un lado, y técnico y vocacional, por el otro. Sus fundamentos eran principalmente psicológicos. Mercante pensó que el principal problema que debían enfrentar las escuelas intermedias y secundarias era "la crisis de la pubertad", y creía que las instituciones educativas tenían que ser rediseñadas para lidiar mejor con ella. Se consideraba a sí mismo un psicólogo -o mejor, un paidólogo, como él definía a su saber-, y aunque nunca obtuvo un diploma universitario, fue el fundador de la primera Escuela de Ciencias de la Educación en la Universidad Nacional de La Plata en 1915. El currículum, para él, debía basarse en la evidencia provista por la psicología. Propuso diferenciar el currículum según el género, y pensó que los adolescentes varones corrían mayor riesgo de "degeneración" que las mujeres. Su insistencia en el trabajo manual estaba fundamentada explícitamente en la necesidad de mantener las manos de los varones ocupadas y visibles (la masturbación era uno de los objetos preferidos del pánico moral de la época). El trabajo manual ocupaba más del 30\% del calendario semanal, y se suponía que serviría para sublimar y disciplinar los instintos sexuales de los varones jóvenes (Mercante leyó a Freud, e incluso lo conoció en Viena en 1911)."

${ }^{2}$ Debemos tener en cuenta que los Colegios Nacionales eran verdaderos representantes de la Nación en las capitales provinciales. Sus majestuosos edificios contrastaban 
los varones de las clases acomodadas, que luego se convertían en figuras de la política y la economía nacional. Es decir, eran escuelas pensadas para una reducida elite. El crecimiento de la clase media (en número y en poder económico), ponía en peligro esta condición de escuela para la elite. Una clase media que podía postergar el ingreso de sus hijos adolescentes al trabajo, presionaba por el ingreso a la escuela media. El triunfo de Hipólito Yrigoyen en 1916, es una clara muestra del avance de estos sectores sociales sobre los espacios reservados tradicionalmente a la elite terrateniente. Tanto la propuesta de Magnasco como la de Saavedra Lamas, incluían caminos alternativos al bachillerato tradicional de los Colegios Nacionales, y de esa manera cerraba el acceso a la universidad. Por aquellos años, solo los egresados del Colegio Nacional tenían acceso a la universidad. A diferencia de lo que sucedía en muchos países europeos3, en la Argentina la idea de una escuela única para todos era muy fuerte. Por tanto, debían buscarse otros caminos para desviar el avance de sectores sociales que no fueran la élite, sobre la escuela media y por consecuencia sobre la universidad. Habilitar el acceso a la escuela secundaria a nuevos sectores sociales derivaría, tarde o temprano, en habilitar el acceso a la universidad.

Si bien las discusiones por la escuela secundaria seguirán dándose durante los años posteriores, a los efectos de este trabajo vamos a elegir la década del '60 como punto partida de nuestros análisis. En esta década, se va dar la que puede considerarse la primera de las reformas integrales del sistema educativo. Esta tendrá una serie de características que volverán a presentarse en las siguientes reformas que tendrán lugar en la Argentina.

con las modestas construcciones de las ciudades del interior del país. Ser alumno del Colegio Nacional implicaba un prestigio social reservado a las elites gobernantes y terratenientes. Estas cuestiones, entre otras, hacía que las clases medias urbanas pujaran porque sus hijos ingresaran al secundario.

3 En líneas generales, en Europa era aceptada la idea de un sistema educativo con dos vías. Una que conducía al mundo académico y otra que preparaba para el mundo del trabajo. En la Argentina la idea de una escuela unificadora (en un contexto de fomento de la inmigración), resultaba innegociable. Los discursos de las figuras públicas no podían omitir este detalle. Quizás por eso, la doble vía tuvo que generarse de modos solapados. 


\section{La década de 1960. La teoría del capital humano y la reforma del ’ 68}

Los años ' 60 son un periodo caracterizado por grandes eventos políticos y sociales que marcaron el rumbo de la historia en occidente. Quizás sea la denominada Guerra Fría la que sirva de contexto para comprender muchos de las decisiones políticas de la época. En el marco de esta contienda no declarada, caben destacar dos hechos que pudieron tener incidencia en las cuestiones que nos ocupan: la puesta en órbita del satélite ruso Sputnik (1957) y la revolución cubana (1959). La exitosa misión rusa que puso en órbita el primer satélite artificial de la historia, puso en jaque al sistema educativo estadounidense. Los soviéticos lograban éxitos en el campo de la ciencia y la tecnología superando a EE.UU. Esta situación generó una fuerte revisión de la educación y en especial de la escuela media. El gobierno estadounidense entendió que debía desechar la educación basada en principios filosóficos y humanista (como la educación propuesta por los escolanovistas) e instalar una educación basada en la ciencia y la tecnología (PICCO, 2014). Había llegado el momento de la teoría curricular (KEMMIS, 1990) y de la psicología conductista (en su versión skinneriana).

Desde principios del siglo XX, había autores abocadas a la formulación de una teoría curricular para la educación. Está teoría proponía una organización científica del currículum escolar basada en técnicas y procedimientos precisos que permitían asegurar el éxito de los objetivos propuestos (JOYCE; WEIL, 2002). Su planteo estaba en clara consonancia con las necesidades políticas del momento. La escuela secundaria estadounidense comenzó a ser reformada a la luz de la nueva teoría curricular norteamericana. La nueva visión pedagógica de la educación prometía logros concretos y medibles por parte del sistema educativo. Por otro lado, la corriente conductista de la psicología estadounidense avanzaba sobre la comprensión del proceso de aprendizaje. Skinner, su máximo exponente, logrará formular una explicación de cómo aprenden las personas. Esta formulación dará lugar a un método de enseñanza que se- 
rá conocido como instrucción programada. Para mediados de los ‘60, los EEUU estaban lanzados de lleno a reformar la escuela media a la luz de la Teoría Curricular de Tyler y la instrucción programada de Skinner.

En el campo de lo económico, comienzan a ganar terreno las ideas desarrollistas, y todas las acciones del Estado se orientan a promover el desarrollo económico. Se instala la Teoría del capital humano, que piensa a la educación como una inversión para lograr el mentado desarrollo. La Teoría del capital humano introduce nuevos términos en el campo de la educación que obligan a repensar la enseñanza (TEDESCO; FANFANI, 2001), (PINEAU, 2006) (BIRGIN, 2007). El sistema educativo debe racionalizar el uso de los recursos y apuntar a objetivos claros y mensurables. Para ello se requiere de una enseñanza basada en técnicas de base científica y con docentes entrenados en la aplicación de esas técnicas.

En 1959, triunfa la revolución cubana (la cual se declara filocomunista a poco de triunfar) en la isla caribeña. A las puertas de los EEUU, se instalaba un enclave de su acérrimo enemigo. Seguramente, este fue uno de los acicates que impulsó a los EE.UU. a extender sus ideas desarrollistas por todo el continente americano. En el caso de la educación, se produjo una verdadera ola de cambio curricular en todo Latinoamérica bajo los auspicios de EE.UU. y de organismos internacionales como la OEA y la UNESCO. Esta es una característica novedosa en temas de reforma educativa. La presencia de los organismos internacionales en las reformas educativas del continente, serán una constante de aquí en más (PINEAU, 2006).

La Argentina no será la excepción en esta ola de cambio curricular (Goodson, 1995). Durante los años 60 las ideas desarrollistas dominarán el escenario económico y también el educativo (FURLÁN; AVOLIO DE COLS, 2011). La teoría curricular norteamericana y la psicología conductista se imponen:

A fines de la década del '6o se comienza a difundir la teoría del currículum, nuestros libros de referencia eran Tyler, Saylor y Alexander e Hilda Taba. Estos autores proponían un modelo de planeamiento que tenía en cuenta las ideas básicas a enseñar, el contexto, destacaban el papel del alumno en la discusión de la propuesta elaborada por el docente y soste- 
nían una concepción de aprendizaje basado en la experiencia. En la década del '70, se comenzó a difundir el modelo tecnológico conductista de planeamiento didáctico. Este fue adoptado por los organismos técnicos del Ministerio de Educación para orientar a los docentes en la implementación de la reforma educativa iniciada en 1971. Comenzó a circular bibliografía de origen estadounidense y mexicano como Chadwick, Briggs, Mager, Gagné, que planteaban un enfoque tecnicista del planeamiento educativo. (FURLÁN; AVOLIO DE COLS, 2011 p. 61).

A pesar de las constantes interrupciones del orden institucional por parte de sectores militares, las ideas desarrollistas (tanto en lo económico como en lo educativo) se mantendrán más o menos constantes a lo largo de todos los gobiernos de la década. En 1968, se sancionará una reforma educativa integral. Es decir, que abarcará todos los niveles del sistema (TIRAMONTI, 2015). Esa reforma será impulsada por la dictadura del General Onganía y acompañada por sectores conservadores del catolicismo (SCIRICA, 2007). Si bien no irá más allá del fin de su gobierno, es interesante rescatar algunas características de la iniciativa reformista y sus fundamentos.

La Resolución 994 de octubre de 1968, sanciona la reforma de todo el sistema educativo argentino. Interesa, para los fines de esta investigación, subrayar los aspectos vinculados a la escuela media. La reforma del '68, crea la escuela intermedia. De alguna manera heredera de las ideas de Saavedra Lamas, esta escuela intermedia se ubica entre la escuela primaria y la secundaria. Se conforma tomando los dos últimos años de la escuela primaria y los dos primeros de la escuela secundaria. La norma fundamenta la creación de este nuevo nivel, básicamente, en los nuevos conocimientos que provee la psicología en relación a las etapas madurativas y de evolución de la inteligencia. Muy a tono con las ideas de la ola curricular en curso. En los motivos que fundamentan la necesidad de esta reforma, los funcionarios citan documentos de la UNESCO y de la CO$\mathrm{NADE}^{4} \mathrm{y}$ otras dependencias del gobierno. En el primer caso, se trata de

\footnotetext{
4 CONADE: Comisión Nacional de Desarrollo. Era un organismo gubernamental que tenía entre sus finalidades orientar las políticas públicas bajo los postulados de las ideas desarrollistas de la época.
} 
documentos que vinculan claramente al sistema educativo con el desarrollo económico de los países subdesarrollados. En el segundo, los organismos nacionales, destacan en distintos informes sobre la educación en el país, que esta está desvinculada del desarrollo del potencial humano impidiendo satisfacer los requerimientos de bienestar de la población.

También menciona que posterga la producción y distribución de bienes y que impide el triunfo de los mejores. La reforma aparece claramente comprometida con las ideas del desarrollo económico y ubica al sistema educativo en consonancia con la teoría del capital humano (CAZAS, 2017). Claramente la norma dice que se buscará racionalizar y hacer más eficiente al sistema educativo para lograr ponerlo al servicio del desarrollo económico de la nación. También resulta claro cuando habla de los sujetos que serán educados en este sistema. Este atenderá a todos los sujetos de acuerdo a sus necesidades. Así atenderá a los alumnos más dotados como aquellos que presenten deficiencias o sean ineptos (en estos términos se expresa la norma). Podría pensarse que al igual que se habla, en esos años, de países desarrollados y países subdesarrollados, también entre los sujetos a educar hay desarrollados y subdesarrollados. Para los primeros habrá que crear las condiciones óptimas para que sigan desarrollándose y para los segundos, habrá que tomar medidas que permitan compensar esas deficiencias que impiden su desarrollo.

Esta reforma rompe claramente con el paradigma del bachillerato francés basado en una formación humanista y de cultura general, para presentarse como una iniciativa que busca modernizar y hacer eficiente al sistema educativo atendiendo a los cambios en el mundo.

\section{El proyecto 13}

A principios de los '70, se sanciona la ley 18614 que será conocida como "Ley del Proyecto 13”. Esta norma había surgido de una iniciativa de un grupo de inspectores de las escuelas nacionales de nivel secunda- 
rio5. Estos inspectores entendían que cualquier intento de reforma al nivel medio debía incluir un cambio en el régimen laboral docente. En el marco de las ideas reformistas de los '60, estos funcionarios habían elaborado una propuesta. Lo central de esta era modificar la tradicional designación por horas cátedra, por una designación por cargo. Esta modificación, que se materializo en la sanción de la ley, implica un reconocimiento económico a las tareas que un profesor desarrolla como parte de su rol, además de dar sus clases. Con este sistema, los profesores pasaron a tener horas rentadas para participar de proyectos institucionales, capacitaciones, talleres extracurriculares, etc.

Esta modificación al régimen laboral de los profesores del nivel medio repercutió en las escuelas dando lugar a la aparición (o bien dándoles mayor impulso) de proyectos institucionales que incidían en forma directa en la mejora de los aprendizajes. A pesar de las evidentes ventajas del nuevo régimen laboral, el Poder Ejecutivo de entonces (otra dictadura militar), limitó su aplicación a un pequeño grupo de escuelas a modo de experiencia piloto. Esta nunca abandonó su condición de piloto y así quedó limitada a ese pequeño grupo de escuelas de todo el país. Es interesante mencionar que las escuelas elegidas, conformaban el núcleo de escuelas públicas a las que asistían los hijos de las clases medias y medias altas urbanas de las grandes capitales del país.

\section{La década del '8o. La pos dictadura y el retorno al sistema democrático}

Desde 1976 hasta 1983, la Argentina estuvo inmersa en la más sangrienta y brutal de las dictaduras. Durante esos años difíciles y oscuros, no hubo grandes novedades en educación en materia de reformas. Los dictadores estuvieron más ocupados purgando a los cuadros docentes y

\footnotetext{
5 El Proyecto 13 no tenía la intención explicita de reformar la escuela media (así lo expresaba la Prof. M. A. Tula que coordinó la comisión encargada de redactar la norma, en una entrevista realizada por el autor de estas líneas en el año 2011), pero el cambio en el régimen laboral docente trajo como consecuencia variaciones en algunos aspectos de la propuesta curricular tradicional.
} 
prohibiendo libros y teorías, que en modernizar el sistema. Así, para los últimos años de la dictadura, ya comenzaban a circular algunos trabajos que subrayaban el deterioro del sistema educativo en general y de la escuela media en particular. Con esos diagnósticos de una escuela secundaria desactualizada (TEDESCO, 1985) y profundamente elitista y autoritaria (BRASLAVSKY, 1985) (KRAWCZYK, 1989), el gobierno constitucional que asumió a fines de 1983 encaró la reformulación de la escuela secundaria argentina.

La demanda de democratización era muy potente en esos años. Esta alcanzaba también al sistema educativo. Durante este periodo no se dará una reforma a través de una norma general que abarque todo el sistema, sino que se irán haciendo cambios paulatinos. En primer lugar, el gobierno constitucional del Dr. Alfonsín, procederá a eliminar el examen de ingreso ${ }^{6}$. Esta prueba que se les tomaba a todos los aspirantes a ingresar a la escuela media y que era de carácter selectivo, es eliminada. De esta forma todos los estudiantes que aspiren a ingresar podrán hacerlo sin ningún requisito más que el de contar con el certificado de aprobación del nivel primario. También se elimina el uso obligatorio de un uniforme escolar, que resultaba un costo importante para las familias de bajos recursos económicos. Uno de los cambios más importantes por su peso en el sistema educativo y en la tradición de la escuela media, es el cambio del régimen de calificación y promoción de los alumnos.

Para el ciclo lectivo 1985, se reemplazan las tradicionales mesas examinadoras de los meses de diciembre por un período de recuperación. Ya no se trata de rendir examen un día determinado frente a un tribunal examinador compuesto por tres profesores, sino que se reemplaza por un curso de apoyo a cargo del profesor del curso. Es decir que de alguna manera, la idea es que para aquellos alumnos que al terminar el año no lograron los aprendizajes esperados, cuenten con una extensión de un mes más de clases para poder alcanzar el rendimiento esperado y así aprobar la materia. Al año siguiente, el ministerio del área promulga una

\footnotetext{
${ }^{6}$ A través de la Resolución 2414 del 29/10/1984 el ministerio de educación y justicia nacional eliminó el requisito del examen de ingreso para la escuela media.
} 
resolución7 que reemplaza al régimen de evaluación, calificación y promoción de los alumnos que estaba vigente desde 1975. Este nuevo régimen, incorpora el sistema de recuperatorio en reemplazo de las mesas examinadoras de diciembre, pero lo más destacado es que reemplaza la escala numérica de 1 a 10 por una escala de tipo conceptual. La nota numérica que se obtenía por promedios es reemplaza por una escala no promediable y que marca si el alumno alcanzó o no los objetivos de aprendizaje de la materia o asignatura.

Sin duda, este cambio fue uno de los más potentes de ese periodo para la escuela secundaria. La formalización de un nuevo régimen de evaluación, calificación y promoción obligaba a un cambio profundo en la forma de pensar la escuela secundaria no solo para los docentes sino para la sociedad en su conjunto. En esta misma línea de cambios, se decide que el alumno que repite un año de cursada, solo debe cursar las materias que desaprobó en lugar de recursar la totalidad de las materias correspondientes a ese año de estudios independientemente de si estaban aprobadas o no. Con relación al régimen laboral docente del Proyecto 13 (que seguía funcionando en las escuelas pilotos), si bien no lo generalizó, retomó su idea al momento de crear el Ciclo Básico General (TIRAMONTI, 2015). La idea era que todas las escuelas medias tuvieran un ciclo básico en común que abarcará los primeros tres años. Los profesores tendrían designación similar a los del Proyecto 13. La experiencia se puso en marcha en un grupo de escuelas pilotos sobre el final del gobierno del Dr. Alfonsín. Está experiencia no se expandió a otras escuelas, pero las que formaban la experiencia piloto siguieron con ella.

Así como la reforma del '68 tenía por objetivo modernizar y racionalizar el sistema educativo para poner al servicio del desarrollo económico, las reformas que se realizaron durante los años '80 tuvieron por meta democratizar el sistema. Todos los cambios realizados en la escuela secundaria estaban en sintonía con la necesidad de democratizarla. Se

\footnotetext{
7 Con la Resolución 136 del 24/2/1986 el ministro nacional del área reemplaza el régimen de evaluación, calificación y promoción de los alumnos de la escuela secundaria por un nuevo régimen.
} 
pretendía que la escuela media formara sujetos participativos y comprometidos con la defensa del orden constitucional y democrático.

\section{La década del ' 90 . Neoliberalismo y mercado}

Durante la década de los '90, américa latina quedó inmersa en la tendencia hacia el neoliberalismo y las economías de mercado, que recorría gran parte de occidente. En la Argentina, se iniciaba un nuevo periodo de gobierno constitucional. Apenas iniciado su mandato, el Dr. Menem, decide la transferencia de la escuela medias de la órbita nacional a la provincial. Esto es un cambio significativo en lo que había sido la tradición del Colegio Nacional ${ }^{8}$. Casi al mismo tiempo, el ministro de educación nacional deroga el régimen de evaluación, calificación y promoción de los alumnos. El nuevo régimen reinstala la escala numérica 1 a 10 y el sistema de promedios para poder promocionar las asignaturas9. Poco tempo después, el gobierno nacional envía al Congreso Nacional una nueva reforma integral del sistema educativo, que será conocida como Ley Federal de Educación (LFE) ${ }^{10}$.

La nueva ley, sancionada en 1993, modifica la estructura del sistema afectando a la escuela secundaria. La escuela primaria pasa a denominarse Escuela General Básica y se conforma en tres niveles de tres años cada uno. Así, la EGB 3 se forma con el último año de la escuela primaria y los dos primeros de la secundaria. La escuela secundaria desaparece y en su lugar se crea el Polimodal de tres años. Este nivel está conformado como un bachillerato con orientaciones. La nueva estructura

8 El pase de jurisdicción de las escuelas nacionales a las provincias determinado por
ley en 1991 provocó una verdadera "herida narcisística" en las comunidades educati-
vas de los Colegios Nacionales. Aún hoy, en muchas capitales provinciales, los frentes
de las escuelas siguen exhibiendo la leyenda "Colegio Nacional"
9 La Resolución $94 / 92$ del $29 / 1 / 1992$ modifica el régimen de evaluación de 1986. La
resolución se redacta a pocos días de la sanción de la "ley de transferencia" de las es-
cuelas medias.
10 La ley 24195 sancionada en 1993 por el Congreso Nacional es la que se conoce co-
mo "Ley federal de educación" 
no contempla a las escuelas de educación técnica, las cuales deben reorientarse a un formato compatible con el de bachiller orientado. Es de destacar que se extiende por primera vez la educación obligatoria (que durante todo el siglo XX se había limitado a la escuela primaria) a diez años incluyendo el último año del nivel inicial y los nueve de la EGB. También se decide realizar una revisión y renovación de los contenidos de la enseñanza. Así surgirán los Contenidos Básicos Comunes (CBC) producto de la consulta a destacados especialistas de cada área del conocimiento convocado por las autoridades ministeriales. Estos nuevos recortes de saberes son presentados como una modernización de los obsoletos contenidos vigentes (en el caso de la escuela media, la mayoría de los programas oficiales databan de las décadas del ' 50 y '60).

Esta reforma educativa integral retomó, en cierto sentido, algunos aspectos dela reforma del '68. Su argumento principal era la modernización del sistema educativo y su puesta al servicio de la economía de mercado. Los organismos internacionales y la Iglesia Católica fueron fuente de consulta para la redacción de la ley y para su posterior implementación. El ministerio de educación y cultura tenía el objetivo explícito de orientar al sistema educativo para promover la competitividad internacional de la economía nacional e incrementar la productividad ${ }^{11}$. Acorde con las políticas económicas reinantes en esos años en gran parte de américa latina, la educación secundaria debía priorizar el desarrollo de competencias en los jóvenes para facilitar su inserción al mercado laboral. Si bien es cierto que en la norma legal se menciona la necesidad de seguir avanzando en la democratización del sistema educativo, estas menciones aparecen más como declaraciones de principios que como intenciones políticas efectivas (PAVIGLIANITI, 1995).

Podría pensarse que la ampliación de la obligatoriedad hasta la finalización de la EGB 3, sería un avance hacia la democratización, sin embargo, es notorio que cuando se leen atentamente los documentos curri-

\footnotetext{
11 Para ampliar estos temas se pueden consultar algunos documentos oficiales de la época como "Aplicación de la Ley Federal de Educación” elaborado por el Ministerio de Cultura y Educación nacional en 1994. Este documento se enviaba a todas las escuelas del país.
} 
culares, los saberes para el acceso a estudios superiores, para el desarrollo de competencias para la inserción laboral y los avances científicos y tecnológicos quedan ubicados por fuera de los niveles obligatorios ${ }^{12}$. Así como algunos vieron en la reforma impulsada por Saavedra Lamas la intención de romper la continuidad lógica que se había instalado entre primaria y secundaria para obstaculizar el acceso a la universidad, podría verse una intención similar al establecer la obligatoriedad de la escuela básica, pero no del polimodal.

Con el pretexto de democratizar el acceso y permanencia en el sistema educativo de todos los jóvenes, se implementaron una serie de políticas compensatorias. El Estado, ubicado nuevamente en un rol subsidiario (dejando el rol principal a las familias y a la Iglesia Católica), debía ocuparse de aquellos jóvenes con carencias. Para estos, el gobierno central desarrolló planes como el denominado Plan Social Educativo (Giovine, 2014) para proveer de recursos a los jóvenes provenientes de sectores sociales con carencias económicas. Así se entregaron útiles escolares, libros de texto, etc. Este mismo plan se extendió a las instituciones escolares que recibían a estas poblaciones de jóvenes, entregando equipamiento y mobiliario.

\section{La crisis del 2001 y una nueva reforma integral}

Hacia fines de la década del '90, la situación económica en la Argentina comenzó a deteriorarse. El previsible colapso de políticas económicas que fomentaban las inversiones financieras que no capitalizaban al país ni fortalecían al aparato productivo, se produjo a fines del 2001. En el 2003, asume un nuevo gobierno constitucional. Este, conducido por el Dr. Kirchner, toma una nueva orientación económica y se aleja del modelo neoliberal que había hegemonizado la escena política en la década anterior. En el año 2005 se sanciona la ley de Educación Técnico Profesio-

12 Ídem anterior. 
nal. Con esta ley se reinstala la educación técnica en la Argentina, que había quedado fuera de estructura del sistema de la Ley Federal de Educación. Al año siguiente, el parlamento deroga la LFE y sanciona la nueva Ley de Educación Nacional (LEN). Esta nueva ley vuelve a la estructura anterior a 1993 conformándose nuevamente un nivel primario (de 607 años) y un nivel secundario (de 5 o 6 años) ${ }^{13}$. Desparece así la EGB 3 .

A diferencia de su antecesora, esta reforma no se funda en las necesidades del aparato productivo del país o en el desarrollo del mercado. Incluso un artículo ${ }^{14}$ prohíbe expresamente que el Estado Nacional firme acuerdo o tratados internacionales de libre comercio que consideren a la educación un bien de mercado. Esta nueva reforma retoma, de alguna manera, los argumentos de democratización e inclusión que sostuvieron los cambios al nivel medio en los '80. La educación es considerara un bien público del cual el Estado es el garante. Esto implica garantizar financiamiento, accesibilidad, inclusión, etc. En esta condición de garante, el gobierno nacional instrumentará una serie de planes y programas tendientes a promover la inclusión de todos los niños y jóvenes a la educación ${ }^{15}$. Estos programas no tendrán la condición de compensatorios (entendiendo esto como la obligación de cubrir aquello que falta) sino que serán políticas pensadas en clave de inclusión. Estas políticas socioeducativas tendrán por objetivo a la totalidad de la población estudiantil pensada como un universo diverso y no como un conjunto homogéneo que incluye sujetos con carencias.

\footnotetext{
13 La ley 26206 es la que se conoce como "Ley de Educación Nacional”. Esta deroga la ley de educación federal y por tanto su estructura. Dada la diversidad de formas en que la ley había sido aplicada en las distintas jurisdicciones, la nueva ley contemplo distintas posibilidades para volver al modelo primaria-secundaria. Por ese motivo algunas jurisdicciones educativas pudieron adoptar el modelo de 6 años de primaria y 6 de secundaria y otras el de 7 años de primaria y 5 de secundaria.

14 Se trata del artículo 10 de la ley.

15 Quizás el ejemplo paradigmático es el ambicioso Plan Conectar-Igualdad que distribuyó alrededor de 3 millones de netbooks a los estudiantes de las escuelas secundarias de todo el territorio nacional.
} 
Una novedad a destacar es que se extiende, por primera vez en la Argentina ${ }^{16}$, la obligatoriedad hasta el nivel medio completo. Es decir que pasa a ser obligatoria la escuela primaria más la escuela secundaria. Esta decisión implica un cambio muy fuerte para una escuela concebida desde sus orígenes como una escuela para las elites gobernantes. Y además generará una presión sobre la universidad, ya que es previsible que aumente el número de egresados de la escuela media. Todo esto obligará a repensar las formas de enseñar en la escuela media, y dará lugar a una nueva reforma del nivel.

Esta fue aprobada por el Consejo Federal de Educación a principios del 2009, a través de dos resoluciones ${ }^{17}$. Con el nombre de Nueva Escuela Secundaria (NES), se delinean una serie de cambios para el nivel medio. Lo interesante de esta reforma es que por primera vez se incluye explícitamente en la norma la revisión de las formas de enseñar. Así se propone revisar la organización institucional de la enseñanza, los regímenes académicos, las regulaciones jurisdiccionales que incidan directamente sobre la enseñanza y las propuestas de enseñanza (las configuraciones de la clase escolar) (CAZAS; DIAMANT, 2016). Las resoluciones del CFE incluyen una serie de propuestas que habilitan nuevas formas de enseñar en la escuela secundaria. De alguna manera, esta reforma parece apuntar a una de las patas del trípode de hierro (TERIGI, 2006) que resiste en la escuela secundaria: la organización del saber en parcelas de conocimientos disciplinar. La consecuencia directa de repensar una nueva organización de los saberes en la escuela media, es revisar las formas del régimen laboral de los profesores. La reforma NES, promueve la generalización del denominado Proyecto 13. La designación de los profesores por cargo facilita la implementación de otras formas de organizar la enseñanza (como talleres, seminarios, etc.). ${ }^{18}$

\footnotetext{
${ }^{16}$ Cabe mencionar la excepción de la Ciudad de Buenos Aires, que estableció la obligatoriedad de la escuela media en su jurisdicción en el año 2002 a través de la ley 898.

17 Las Resoluciones 84 y 93 del Consejo Federal de Educación del año 2009 son la base normativa de la denominada Nueva Escuela Secundaria (NES)

18 En la Ciudad de Buenos Aires se ha generalizado la designación por cargo a partir de la sanción de una ley de la ciudad del año 2009.
} 


\section{Fuerzas y tensiones en las propuestas reformadoras}

Si observamos con cierto detenimiento esta serie de propuestas e iniciativas de reformas del nivel medio o secundario, podemos encontrar similitudes y diferencias entre ellas. Si miramos las propuestas de los ' 60 y de los '90, podemos identificar algunos puntos en común: la creación de un nivel intermedio que se ubica entre la primaria y la secundaria; la universalización del acceso en el nivel de la escuela primaria o básica; el propósito de orientar el nivel medio hacia el desarrollo económico y del mercado; la idea de una escuela que busque y "premie" a los mejores; acciones para ayudar a los estudiantes carenciados. Si observamos las iniciativas de reformas de los ' 80 y los 2000 podremos ver coincidencias en: el mantenimiento del esquema escuela primaria y secundaria (esta como paso previo al acceso a la universidad); la tendencia a universalizar el acceso tanto a la escuela primaria como a la secundaria completa; el propósito de orientar a la escuela secundaria hacia el fortalecimiento de la ciudadanía y de las instituciones democráticas; la idea de una escuela secundaria para todos con políticas inclusivas sociales y educativas.

En cierta medida podríamos pensar que el período que va de 1984 al 2010 está atravesado por una cantidad importante de iniciativas reformistas (que afectaron a la escuela secundaria en particular), que están signadas por una tensión entre dos fuerzas: una democratizante y otra aristocratizante (CAZAS, 2015). No es que se pueda encontrar una reforma en la cual solo esté presente una fuerza o la otra, sino que ambas pujan en cada iniciativa por imponerse sobre la otra. Como sucede con las fuerzas en la Física, las reformas que finalmente se implementan son la resultante de estas dos fuerzas concurrentes en un punto: la escuela media. Las reformas de los '80 y los 2000 tienen un fuerte predominio de la fuerza democratizadora, pero esto no significa que no sobrevivan en ellas aspectos aristocratizantes. Lo mismo sucede si tomáramos las reformas de los ‘9o (o su antecesora de los ‘60). Tienen un predominio de la fuerza aristocratizante pero ello no quita la presencia de elementos de la fuerza 
democratizante. En cierto modo la escuela secundaria terminaría siendo la resultante de esta tensión manifiesta en cada proceso de reforma.

¿Por qué hablar de fuerza democratizante y fuerza aristocratizante? Por democratizante me referiré a la fuerza reformadora que plantea acceso, permanencia y egreso de la escuela media para todos. Con aristocratizante me referiré a esa fuerza reformadora que piensa a la escuela media como una escuela para los que se la merecen. Es decir, que el acceso a la escuela secundaria debe ser circunscripto solo a los mejores, a los que tienen mérito suficiente para estar en ella. Podría utilizar la expresión meritocratizante, sin embargo, ese término dejaría por fuera una cuestión sustancial. La fuerza aristocratizante piensa en términos de méritos, pero asociada a origen social. Esta fuerza añora los tiempos del Colegio Nacional para las élites gobernantes y terratenientes. Por ese motivo me parece más adecuado usar el término aristocratizante, ya que refiere a la meritocracia sumada al origen de clase social. Por otra parte, pensarlo en términos de fuerzas concurrentes (haciendo esta analogía con la Física) permite una representación muy gráfica de la situación.

Dos fuerzas que actúan sobre un mismo punto, cuyo efecto sobre este no responde enteramente a una fuerza o a la otra. Sino que se trata de una resultante que contiene, en cierto modo, un poco de ambas. La escuela secundaria, en cada momento reformista, parece ser la resultante de la tensión a que ambas fuerzas la someten. Es decir que en los '90, las escuelas medias no estaban absolutamente alineadas con la fuerza aristocratizante, en todo caso esta predominaba por sobre la otra democratizante. Como sostienen varios autores (EZPELETA, 2004; VIÑAO FRAGO, 2003), para implementar innovaciones en la escuela no basta una norma legal. Cada iniciativa reformista debe lidiar con las tradiciones, con los mandatos fundacionales, con la cultura escolar, en síntesis, con la memoria de las escuelas. Por eso no podemos pensar en términos absolutos cuando se habla de reformas en las escuelas medias. 


\section{Los niveles de}

\section{acción de las reformas}

El accionar de las reformas del sistema educativo puede pensarse en tres niveles. Hay un primer nivel que tienen que ver con el gobierno del sistema; un segundo nivel que refiere al gobierno de las instituciones y sus formatos; un tercer nivel que refiere a las prácticas de enseñanza que se suceden en el cotidiano de la tarea escolar. Algunas reformas hacen más hincapié en uno u otro nivel. La reforma de los 90 puso mucho énfasis en las reformas en el nivel de gobierno del sistema y en el nivel de gobierno de las instituciones. Las reformas de los '80 y de los 2000 pusieron más énfasis en el nivel de las prácticas de enseñanza. Por supuesto que unas y otras no descuidaron el resto de los niveles, pero hay un especial énfasis en un u otro nivel según de que reforma se trate. Visto de esta manera pareciera que unas reformas han sido pensadas como algo que se efectúa desde la cima del gobierno del sistema hacia la base (en una visión piramidal del sistema), y otras ven a la reforma como algo que debe arrancar desde la base y subir hacia la cima.

Estas dos visiones son muy compatibles con la definición que ya hemos hecho de una fuerza democratizante y una aristocratizante. Pareciera ser que en el caso de las primeras se ve a las reformas iniciándose desde la base y en cambio las segundas lo ven iniciándose desde arriba. Esta mirada está en consonancia con análisis hechos por algunos autores sobre la reforma del '90 (PAVIGLIANITI, 1995). No obstante, el énfasis puesto en uno u otro nivel, todas tiene una posición tomada con relación a las configuraciones de la clase escolar. Hay prácticas de enseñanza compatibles con las reformas aristocratizantes y hay otras compatibles con las democratizantes. 


\section{Prácticas de}

\section{enseñanza y reformas}

Como ya dijimos, cada tipo de reformas tienen unas prácticas de enseñanza que le son funcionales a sus propósitos. Aun cuando la norma no las mencione explícitamente. Las reformas con predominio de la fuerza aristocratizante, apuntan a unas prácticas de enseñanza dirigidas a un sujeto único. De alguna manera, se piensa a los sujetos de la educación media en la misma clave que las ideas desarrollistas piensan a los países. Hay países desarrollados y otros en desarrollo. Es decir que hay un modelo de país al que todos quieren alcanzar. Los países en desarrollo deben ser ayudados por los desarrollados para que puedan ser como ellos. Así es pensado el sujeto en estas reformas. Hay jóvenes con méritos propios que son merecedores de acceder a la escuela media (normalmente proveniente de sectores medios altos y altos). Para aquellos que tienen carencias o deficiencias (los ineptos los llamaba la reforma del '68) el sistema educativo debe generar políticas compensatorias. ¿Qué compensan las políticas compensatorias? Compensan la falta, la carencia de ciertas aptitudes que tienen algunos estudiantes en relación al modelo a alcanzar. Es decir, que podría pensarse en unas prácticas de enseñanza que tienen un estudiante modelo o ideal (generalmente el joven de clase media alta $o$ alta hijo de profesionales o de terratenientes) y que con cierta actitud piadosa se encarga de compensar las carencias de aquellos que no alcanzan el ideal.

Esto tiene fuertes implicancias a la hora de las prácticas de enseñanza. Si hay un estudiante modelo, la forma de enseñar es aquella en la que ese joven aprende. Un modelo de alumno, un modelo de enseñanza. Quienes no pueden aprender en ese modelo de enseñanza es a consecuencia de sus carencias. Los profesores más piadosos se inclinarán por la aplicación de los programas y planes compensatorios y los menos opinarán que ese joven "no es para la escuela secundaria”. Aquí es válido recordar las quejas de los docentes que definen a los estudiantes en términos de sus carencias: las familias no se ocupan, vienen de hogares po- 
bres, no muestran interés por lo que se les explica, etc. En estos comentarios subyace como modelo las prácticas de enseñanza solidarias con una reforma de predomino aristocratizante. En las reformas de corte democratizante, las prácticas de enseñanzas que resultan en línea con estas son aquellas que no parten de la idea de un estudiante modelo al que todos deben tratar de alcanzar.

Estas prácticas de enseñanza parten de reconocer una diversidad de modelos de estudiante y por tanto generan una variedad de formas de enseñar. Sin estudiante único, no hay modelo único de enseñanza. Un modelo plural de estudiantes fundamenta una pluralidad de formas de enseñar. Aquí podría reconocerse a esos profesores que plantean cuestiones del estilo: si no aprenden de una manera pruebo con otra; si no les fue bien con esta prueba, diseño otra con distinto formato, etc. Estas prácticas de enseñanza son claramente inclusivas y buscan reconocer a cada estudiante en su diversidad y distribuir el conocimiento de manera equitativa y no con criterios meritocráticos.

Como puede verse, en el nivel de las prácticas de enseñanza también puede reconocerse la presencia de una fuerza aristocratizante y una democratizante en los términos definidos antes. En cierto modo las prácticas de enseñanza (las configuraciones del dispositivo clase escolar) también pueden ser interpretadas como la resultante de la tensión entre una fuerza democratizante y otra aristocratizante. Así las prácticas de enseñanza se configurarán dentro del amplio espectro de resultantes que produce la tensión entre ambas fuerzas.

\section{Referencias}

BIRGIN, A. (2007) Pensar la formación de los docentes de nuestro tiempo. En TERIGI (comp) Diez miradas sobre la escuela primaria. Buenos Aires. Fundación OSDE/Siglo XXI 
BRASLAVSKY, C. (1985) La discriminación educativa en Argentina, Buenos Aires, Miño y Dávila.

CAZAS. F. (2017) Un encuentro complejo entre el Psicoanálisis y el Conductismo. En Revista Espacios en Blanco. Tandil. UNC. Aprobado para su publicación en el Nro. 27 de Junio 2017.

. (2015) Moebius o la ilusión de una escuela secundaria para todos. En Multiárea Revista de Didáctica. Castilla - La Mancha. UCLM. Nro. 7, pp 167-182.

CAZAS, F.; DIAMANT, A.; DUHALDE, M (2016) Formación docente, traza didáctica y subjetividad. En Anuario de Investigaciones (Fac. de Psicología de la UBA), vol. 22, p. 99-106.

DUSSEL, I. (2006) Currículum y conocimiento en la escuela media Argentina. En Anales de la educación común. La Plata. Dirección General de Cultura y Educación de la Provincia de Buenos Aires. Tercer siglo, año 2, número 4 / Filosofía política del currículum, p. 95-105.

EZPELETA, J. (2004) Innovaciones educativas. En Revista Mexicana de Investigación Educativa, vol. 9, n. 21, p. 403-424.

FURLÂN, A.; AVOLIO DE COLS, S. (2011) El campo de las Ciencias de la Educación en la trayectoria de los actores: Entrevistas a Alfredo Furlán y Susana Avolio de Cols Archivos de Ciencias de la Educación, 4a. época, 5(5). Disponible en Memoria Académica: http://www.memoria.fahce. unlp.edu.ar/art_revistas/pr.5428/pr.5428.pdf. Acceso en: 11 Jul. 2017.

GIOVINE, R.; MARTIGNONI, L. (2014) Lo socioeducativo y la tensión libertad/seguridad en las políticas y prácticas de inclusión educativa. En: CORBALÁN. (comp.) La cultura al poder. Red de educación, cultura y política en América Latina. Buenos Aires. Biblos.

GOODSON, I. (1995) Historia del currículum. La construcción social de las disciplinas escolares. Barcelona. Pomares Corredor.

JOYCE, B.; WEIL, M. (2002). Modelos de enseñanza. Barcelona: Gedisa. PAVIGLIANITI, N. (1995) La ley federal de educación como elemento de regulación de la realidad educacional argentina. Buenos Aires. OPFFyL. PICCO, S. (2014) Concepciones en torno a la normatividad en la didáctica. Un análisis interdisciplinario de obras teóricas didácticas y curriculares en la Argentina, entre 1960 y 1990; Tesis de Maestría en Ciencias de la Educación, Universidad Nacional de La Plata, Facultad de Humanidades y Ciencias de la Educación, Disponible en: http://www.memoria. fahce.unlp.edu.ar/tesis/te.990/te.990.pdf. Acceso en: Jun. 2015

PINEAU, P. y otros (2006) El principio del fin. Políticas y memorias de la educación en la última dictadura militar (1976-1983). Ed. Colihue. Buenos Aires. Parte I punto II. 
KEMMIS, S. (1990). Introducción. En Carr, W. Hacia una ciencia crítica de la educación (p.7-38). Barcelona: Laertes.

KRAWCZYK, N. (1989) Los procesos institucionales y la discriminación educativa en los colegios secundarios argentinos. En Propuesta Educati$v a$, año $\mathrm{I}, \mathrm{n}^{\circ} 1$.

SCIRICA, E. (2007) Educación y guerra contrarrevolucionaria. Una propuesta de Ciudad Católica-Verbo. Clio \& asociados. Revista de la Fac. de Humanidades de la UNLP. Número 11.

TEDESCO, J. C.; TENTI FANFANI, E. (2001) La reforma educativa en la Argentina. Semejanzas y particularidades. Buenos Aires. IIPEUNESCO.

TEDESCO, J. C.; BRASLAVSKY, C.; CARCIOFI, R. (1985) El Proyecto Educativo Autoritario. Argentina 1976-1982. Buenos Aires, FLACSO GEL.

TERIGI, F. (2006): Los cambios en el currículo de la escuela secundaria ¿Por qué son tan difíciles? Disponible en: http://unesdoc.unesco.org/ images/o015/o01516/151698s.pdf\#151692. Acceso en: 11 Jul. 2017.

TIRAMONTI, G. (2015) La reforma de la escuela media: un anhelo frustrado en Revista Propuesta Educativa. FLACSO, n. 44, año 24, vol. 2, p. 24-37.

TIRAMONTI, G.; SUASNABAR, C. (2015) La reforma educativa nacional en busca de una interpretación. En Revista Aportes para el Estado y la Administración Gubernamental. Disponible en: http://www.asociacionag.org.ar/pdfaportes/15/a15_o9.pdf. Acceso en: 11 Jul. 2017.

VINAO FRAGO, A. (2003) Sistemas Educativos, Culturas Escolares y Reformas. Madrid, Morata, p. 32-34. 